\title{
Some remarks on the orthogonality of generalized eigenfunctions for singular second-order differential equations
}

\author{
By
}

\section{Yoshimi SaItō}

Let us consider a differential equation of second order

$$
\frac{d^{2} u}{d x^{2}}-q(x) u(x)+\lambda u(x)=0, \quad(0<x<\infty) .
$$

Here $q(x)$ is a real-valued function which is locally summable in $(0, \infty)$.

In the case where $x=0$ is a regular point of the equation, M. Matsuda has proved that any pseudo-spectral measure in the limit point case at $x=\infty$ is the Weyl spectral measure (Matsuda [6]).

In this paper we try to extend this result to the case where $x=0$ may be a singular point of the equation.

We take a linearly independent system of solutions $\left(\varphi_{1}(x, l), \varphi_{2}(x, l)\right)$ of the equation $(0.1)$ which satisfies

$$
\phi_{i}(1, l)=\eta_{i}(l), \quad \frac{\partial \varphi_{i}}{\partial x}(1, l)=\zeta_{i}(l)
$$

for $i=1,2$ where $\eta_{i}(l)$ and $\zeta_{i}(l)$ are entire functions of $l$ which satisfy $\eta_{2}(l) \zeta_{1}(l)-\eta_{1}(l) \zeta_{2}(l)=1$ for every complex number $l$.

M.H. Stone, E.C. Titchmarsh and K. Kodaira proved that there exists a spertral measure matrisx $\boldsymbol{P}(\lambda)=\left(\rho_{i j}(\lambda)\right)_{i, j=1,2}$ which satisfies the following three conditions (Kodaira [3], [4]):

(A) $\boldsymbol{P}(\lambda)$ is a positive semi-definite measure matrix on $(-\infty, \infty)$.

(B) Denote by $\mathrm{L}_{2}(-\infty, \infty ; d \boldsymbol{P}(\lambda))$ the Hilbert space with the norm

$$
\|\boldsymbol{v}\|^{2}=\int_{-\infty}^{\infty} \widetilde{\boldsymbol{v}}(\lambda) d \boldsymbol{P}(\lambda) \overline{\boldsymbol{v}}(\lambda),
$$

where $\boldsymbol{v}(\lambda)$ is a vector-valued function on $(-\infty, \infty)$ 


$$
\boldsymbol{v}(\lambda)=\left(\begin{array}{l}
v_{1}(\lambda) \\
v_{2}(\lambda)
\end{array}\right),
$$

$\tilde{\boldsymbol{v}}(\lambda)$ is the transpose of $\boldsymbol{v}(\lambda)$, and $\bar{\alpha}$ means the conjugate complex number of $\alpha$. Then the generalized Fourier transformation

$$
\mathscr{F}_{\boldsymbol{P}}: f(x) \rightarrow \int_{0}^{\infty} f(x) \boldsymbol{y}(x, \lambda) d x, \quad \boldsymbol{y}(x, l)=\left(\begin{array}{c}
\varphi_{1}(x, l) \\
\varphi_{2}(x, l)
\end{array}\right)
$$

from $\mathrm{L}_{2}(0, \infty ; d x)$ into $\mathrm{L}_{2}(-\infty, \infty ; d \boldsymbol{P}(\lambda))$ is isometric.

(C) $\mathscr{I}_{\boldsymbol{P}}$ transforms $\mathrm{L}_{2}(0, \infty ; d x)$ onto $\mathrm{L}_{2}(-\infty, \infty ; d \boldsymbol{P}(\lambda))$.

In Theorem 1 we shall prove that if both $x=0$ and $x=\infty$ belong to the limit point case, then the measure matrix which satisfies (A), (B) and (C) is unique.

We shall prove in Theorem 2 that if both $x=0$ and $x=\infty$ belong to the limit point case, then any measure matrix which satisfies (A) and (B) satisfies (C).

Aknowledgements. I took up this problem, suggested by Mr. M. Matsuda and had a lot of useful discussions with him in the course of writing this paper. I would like to express my gratitude.

\section{$\S 1$ The measure matrix in the eigenfunction expansion for singular differential equations.}

Let us consider a differential equation of the second order

$$
\frac{d^{2} u}{d x^{2}}-q(x) u(x)+\lambda u(x)=0, \quad(0<x<\infty),
$$

where $q(x)$ is a locally summable function in $(0, \infty)$. We assume that $x=0$ is a singular point of the equation. Moreover we assume that the equation (1.1) is of the limit point type both at 0 and at $\infty$.

Let $\left(\varphi_{1}(x, l), \varphi_{2}(x, l)\right)$ be a linearly independent system of solutions of (1.1) which satisfies

$$
\varphi_{i}(1, l)=\eta_{i}(l), \quad \frac{\partial \varphi_{i}}{\partial x}(1, l)=\zeta_{i}(l)
$$

for $i=1,2$, where $\eta_{i}(l)$ and $\zeta_{i}(l)$ are entire functions of $l$ such that

$$
\eta_{2}(l) \zeta_{1}(l)-\eta_{1}(l) \zeta_{2}(l)=1
$$


for every complex number $l$. Then for $\left(\varphi_{1} \varphi_{2}\right)$ there exists a matrix function on $(-\infty, \infty)$

$$
\boldsymbol{P}(\lambda)=\left(\begin{array}{ll}
\rho_{11}(\lambda) & \rho_{12}(\lambda) \\
\rho_{21}(\lambda) & \rho_{22}(\lambda)
\end{array}\right), \quad \rho_{12}(\lambda) \equiv \rho_{21}(\lambda),
$$

which satisfies the following three conditions (A), (B) and (C) (Kodaira [3]):

(A) Each $\rho_{i j}(\lambda)$ is a function of bounded variation on every finite interval in $(-\infty, \infty)$, and $\boldsymbol{P}(\lambda)$ is a positive semi-definite measure on $(-\infty, \infty)$. Namely for every finite interval $\Delta$ and for every pair of continuous functions

$$
v_{0}(\lambda)=\left(\begin{array}{l}
v_{1}^{0}(\lambda) \\
v_{2}^{0}(\lambda)
\end{array}\right)
$$

we have the inequality

$$
\int_{\Delta} \tilde{\boldsymbol{v}}_{0}(\lambda) d \boldsymbol{P}(\lambda) \overline{\boldsymbol{v}}_{0}(\lambda)=\sum_{i, j=1,2} \int_{\Delta} v_{i}^{0}(\lambda) \bar{v}_{j}^{0}(\lambda) d \rho_{\imath j}(\lambda) \geqq 0,
$$

where $\tilde{\boldsymbol{v}}_{0}(\lambda)$ is the transpose of $\boldsymbol{v}_{0}(\lambda)$.

(B) The generalized Fourier transformation from $\mathrm{L}_{2}(0, \infty ; d x)$ into $\mathrm{L}_{2}(-\infty, \infty ; d \boldsymbol{P}(\lambda))$

$$
\mathscr{I}_{\boldsymbol{P}}: f(x) \rightarrow \int_{0}^{\infty} f(x) \boldsymbol{y}(x, \lambda) d x, \quad \boldsymbol{y}(x, l)=\left(\begin{array}{c}
\varphi_{1}(x, l) \\
\varphi_{2}(x, l)
\end{array}\right)
$$

is isometric. Here the element of $\mathrm{L}_{2}(-\infty, \infty ; d \boldsymbol{P}(\lambda))$ is a pair of measurable functions

$$
\boldsymbol{v}(\lambda)=\left(\begin{array}{l}
v_{1}(\lambda) \\
v_{2}(\lambda)
\end{array}\right)
$$

such that

$$
\|\boldsymbol{v}(\lambda)\|^{2} \equiv \int_{-\infty}^{\infty} \tilde{\boldsymbol{v}}(\lambda) d \boldsymbol{P}(\lambda) \overline{\boldsymbol{v}}(\lambda)<\infty .
$$

(C) $\mathscr{I}_{\boldsymbol{P}}$ transforms $\mathrm{L}_{2}(0, \infty ; d x)$ onto $\mathrm{L}_{2}(-\infty, \infty ; d \boldsymbol{P}(\lambda))$.

We shall prove the following two theorems.

Theorem 1. Let the equation (1.1) be of the limit point type both at 0 and at $\infty$. Then the measure matrix which satisfies (A), (B) and (C) is uniquely determined by $\boldsymbol{y}(x, l)$. 
Theorem 2. Let the equation (1.1) be of the limit point type both at 0 and at $\infty$. Then if a measure matrix satisfies (A) and (B) with respect to $\boldsymbol{y}(x, l)$, then it satisfies $(\mathrm{C})$.

To prove Theorem 1 and Theorem 2 we prepare the following lemma.

Lemma 1. Let $\boldsymbol{P}(\lambda)$ be a measure matrix which only satisfies (A) and $(\mathrm{B})$ with respect to $\boldsymbol{y}(x, l)$ and put

$$
E_{P}(x, y ; \Delta)=\int_{\Delta} \tilde{\boldsymbol{y}}(x, \lambda) d \boldsymbol{P}(\lambda) \boldsymbol{y}(y, \lambda) .
$$

Then

(i) $E_{P}(x, y ; \Delta)$ is a symmetric kernel of Carleman type such that

$$
\int_{0}^{\infty}\left(E_{\boldsymbol{P}}(x, y ; \Delta)\right)^{2} d x \leqq \int_{\Delta} \tilde{\boldsymbol{y}}(y, \lambda) d \boldsymbol{P}(\lambda) \boldsymbol{y}(y, \lambda),
$$

and

$$
\int_{0}^{\infty} E_{\boldsymbol{P}}(x, y ; \Delta) f(y) d y=\int_{\Delta} \tilde{\mathscr{F}}_{\boldsymbol{P}} f(\lambda) d \boldsymbol{P}(\lambda) \boldsymbol{y}(x, \lambda)
$$

hold for every $f(x)$ in $\mathrm{L}_{2}(0, \infty ; d x)$.

(ii) Let $E_{P}(\Delta)$ be a linear transformation defined by

$$
E_{P}(\Delta) f(x)=\int_{0}^{\infty} E_{P}(x, y ; \Delta) f(y) d y
$$

for $f(x)$ in $\mathrm{L}_{2}(0, \infty ; d x)$. Then $E_{P}(\Delta)$ is a bounded symmetric operator on $\mathrm{L}_{2}(0, \infty ; d x)$ and we have

$$
\begin{gathered}
\left\|E_{P}(\Delta)\right\| \leqq 1, \quad \lim _{\Delta \rightarrow(-\infty, \infty)} E_{P}(\Delta)=\text { identity }, \\
\left\langle E_{P}(\Delta) f, u\right\rangle=\int_{\Delta} \tilde{\mathscr{F}}_{P} f(\lambda) d \boldsymbol{P}(\lambda) \overline{\mathscr{F}}_{P} u(\lambda)
\end{gathered}
$$

for every pair of $f(x), u(x)$ in $\mathrm{L}_{2}(0, \infty ; d x)$.

(iii) For $y$ fixed $\frac{\partial E_{P}(x, y ; \Delta)}{\partial y}$ belongs $\mathrm{L}_{2}(0, \infty ; d x)$ and we have

$$
\int_{0}^{\infty}\left(\frac{\partial E_{\boldsymbol{P}}(x, y ; \Delta)}{\partial y}\right)^{2} d x \leqq \int_{\Delta} \frac{\partial \widetilde{\boldsymbol{y}}(y, \lambda)}{\partial y} d \boldsymbol{P}(\lambda) \frac{\partial \boldsymbol{y}(y, \lambda)}{\partial y}
$$

In the case where $x=0$ is a regular point, we have a corresponding fact as follows: 
Lemma 2. Let $\rho(\lambda)$ be a speudo-spectral measure ${ }^{(1)}$ for the equation

$$
\frac{d^{2} u}{d x^{2}}-q(x) u(x)+\lambda u(x)=0, \quad(0 \leqq x<\infty),
$$

with respect to the solution $\varphi(x, l)$ and put

$$
E_{\rho}(x, y ; \Delta)=\int_{\Delta} \varphi(x, \lambda) \varphi(y, \lambda) d \rho(\lambda),
$$

where $\Delta$ is a finite interval and $x, y \geqq 0$. Then

(i) $E_{\mathrm{\rho}}(x, y ; \Delta)$ is a bounded symmetric kernel of Carleman type such that

$$
\int_{0}^{\infty}\left(E_{\rho}(x, y ; \Delta)\right)^{2} d x \leqq \int_{\Delta} \varphi^{2}(y, \lambda) d \rho(\lambda),
$$

and

$$
\int_{0}^{\infty} E_{\rho}(x, y ; \Delta) f(y) d y=\int_{\Delta} \varphi(x, \lambda) \mathscr{F}_{\rho} f(\lambda) d \rho(\lambda)
$$

hold for $f(x)$ in $\mathrm{L}_{2}(0, \infty ; d x)$.

(ii) Let $E_{\mathrm{\rho}}(\Delta)$ be a linear transformation defined by

$$
E_{\rho}(\Delta) f(x)=\int_{0}^{\infty} E_{\rho}(x, y ; \Delta) f(y) d y
$$

for $f(x)$ in $\mathrm{L}_{2}(0, \infty ; d x)$. Then $E_{\rho}(\Delta)$ is a bounded symmetric operator on $\mathrm{L}_{2}(0, \infty ; d x)$ and we have

$$
\begin{gathered}
\left\|E_{\rho}(\Delta)\right\| \leqq 1, \quad \lim _{\Delta \rightarrow(-\infty, \infty)} E_{\rho}(\Delta)=\text { identity, } \\
\left\langle E_{\rho}(\Delta) f, u\right\rangle=\int_{\Delta} \mathscr{T}_{\rho} f(\lambda) \overline{\mathscr{F}}{ }_{\rho} u(\lambda) d \rho(\lambda)
\end{gathered}
$$

for every pair of $f(x), u(x)$ in $\mathrm{L}_{2}(0, \infty$; $d x)$.

(iii) For $y$ fixed, $\frac{\partial E_{\rho}(x, y ; \Delta)}{\partial y}$ belongs to $\mathrm{L}_{2}(0, \infty ; d x)$ and we have

$$
\int_{0}^{\infty}\left(\frac{\partial E_{\rho}(x, y ; \Delta)}{\partial y}\right)^{2} d x \leqq \int\left(\frac{\partial \varphi(y \lambda)}{\partial y}\right)^{2} d \rho(\lambda) .
$$

We only prove Lemma 2, because the proof of Lemma 1 is similar.

Proof of (i). Consider a linear functional on $\mathrm{L}_{2}(0, \infty ; d x)$

(1) Matsuda [6]. 


$$
l_{x_{0}, \Delta}(f)=\int_{\Delta} \varphi\left(x_{0}, \lambda\right) \mathscr{F}_{\rho} f(\lambda) d \rho(\lambda)
$$

for $x_{0}$ and $\Delta$ fixed. Then we have

$$
\text { (1.14) } \begin{aligned}
\left|l_{x_{0}, \Delta}(f)\right| & \leqq\left[\int_{\Delta} \varphi_{2}\left(x_{0}, \lambda\right) d \rho(\lambda)\right]^{1 / 2}\left[\int_{\Delta}\left|\mathscr{F}_{\rho} f(\lambda)\right|^{2} d \rho(\lambda)\right]^{1 / 2} \\
& \leqq\left[\int_{\Delta} \varphi^{2}\left(x_{0}, \lambda\right) d \rho(\lambda)\right]^{1 / 2}\left\|\mathscr{F}_{\rho} f\right\|_{\rho} \\
& =M_{x_{0}, \Delta}\|f\|, \quad\left(M_{x_{0}, \Delta}=\left[\int_{\Delta} \varphi^{2}\left(x_{0}, \lambda\right) d \rho(\lambda)\right]^{1 / 2}\right) .
\end{aligned}
$$

This shows that $l_{x_{0}, \Delta}$ is a bounded linear functional. And hence by Riesz theorem we can find a function $e_{x_{0}, \Delta}(x)$ in $\mathrm{L}_{2}(0, \infty ; d x)$ such that

$$
l_{x_{0}, \Delta}(f)=\int_{\Delta} \varphi\left(x_{0}, \lambda\right) \mathscr{F}_{\rho} f(\lambda) d \rho(\lambda)=\int_{0}^{\infty} e_{x_{0}, \Delta}(x) f(x) d x .
$$

On the other hand we have

$$
l_{x_{0}, \Delta}\left(f_{0}\right)=\int_{0}^{\infty} E\left(x_{0}, x ; \Delta\right) f_{0}(x) d x
$$

for $f_{0}(x)$ in $\mathrm{L}_{2}(0, \infty ; d x)$ which has a compact carrier. It follows from (1.15) and (1.16) that

$$
E\left(x_{0}, x ; \Delta\right)=e_{x_{0}, \Delta}(x),
$$

and hence $E(x, y ; \Delta)$ is a kernel of Carleman type and we have (1.10) by (1.15) and (1.16). (1.9) follows from (1.14).

Proof of (ii). Putting $f_{\Delta}(x)=E_{\rho}(\Delta) f(x)$, we have

$$
\begin{aligned}
\left|\int_{0}^{\infty} f_{\Delta}(x) \overline{u(x)} d x\right| & =\left|\int_{0}^{\infty}\left[\int_{\Delta} \varphi(x, y) \mathscr{F}_{\rho} f(\lambda) d \rho(\lambda)\right] \overline{u(x)} d x\right| \\
& =\left|\int_{\Delta} \mathscr{F}_{\rho} f(\lambda) \overline{\mathscr{F}_{\rho} u(\lambda)} d \rho(\lambda)\right| \\
& \leqq\left[\int_{\Delta}\left|\mathscr{F}_{\rho} f(\lambda)\right|^{2} d \rho\right]^{1 / 2}\left[\int_{\Delta}\left|\mathscr{F}_{\rho} u(\lambda)\right|^{2} d \rho\right]^{1 / 2} \\
& \leqq\|f\|\|u\|
\end{aligned}
$$

for $u(x)$ in $\mathrm{L}_{2}(0, \infty ; d x)$ which has a compact carrier. For a positive $N$ and a finite interval $\Delta$, let us define $u_{N, \Delta}(x)$ by

$$
u_{N, \Delta}(x)= \begin{cases}f_{\Delta}(x), & 0 \leqq x \leqq N \\ 0, & x>N\end{cases}
$$


Then $u_{N, \Delta}(x)$ belongs to $\mathrm{L}_{2}(0, \infty ; d x)$ and so (1.17) implies

$$
\int_{0}^{N}\left|f_{\Delta}(x)\right|^{2} d x \leqq\left[\int_{0}^{N}\left|f_{\Delta}(x)\right|^{2} d x\right]^{1 / 2}\|f\|,
$$

namely

$$
\left[\int_{0}^{N}\left|f_{\Delta}(x)\right|^{2} d x\right]^{1 / 2} \leqq\|f\| .
$$

Since $N$ is arbitrary, (1.18) implies

$$
\left\|E_{\rho}(\Delta) f\right\| \leqq\|f\| \text {. }
$$

Proof of (iii). Consider a linear functional on $\mathrm{L}_{2}(0, \infty ; d x)$

$$
k_{x_{0}, \Delta}(f)=\int_{\Delta} \frac{\partial \varphi\left(x_{0}, \lambda\right)}{\partial x} \mathscr{F}_{\rho} f(\lambda) d \rho(\lambda)
$$

for $x_{0}$ and $\Delta$ fixed. Then we have

$$
\text { (1.19) }\left|k_{x_{0}, \Delta}(f)\right| \leqq \tilde{M}_{x_{0}, \Delta}|| f||, \quad\left(\left(\tilde{M}_{x_{0}, \Delta}=\left[\int_{\Delta}\left(\frac{\partial \varphi\left(x_{0}, \Delta\right.}{\partial x}\right)^{2} d \rho(\lambda)\right]^{1 / 2}\right)\right.
$$

as in the proof of (i). By the method used in the proof of (i), we can show that $\frac{\partial E_{\rho}(x, y ; \Delta)}{\partial y}$ belongs to $\mathrm{L}_{2}(0, \infty ; d x)$ and that (1. 12) holds.

Proof of Theorem 1. Let $\boldsymbol{P}_{1}(\lambda)$ and $\boldsymbol{P}_{2}(\lambda)$ satisfy (A), (B) and (C). We shall denote by $\mathscr{D}_{\infty}$ the space of all functions $u(x)$ in $\mathrm{L}_{2}(0, \infty ; d x)$ that satisfy the following conditions:

i) $u(x) \in \mathrm{L}_{2}(0, \infty ; d x)$.

ii) $u(x)$ is differentiable in the open interval $(0, \infty)$.

iii) $\frac{d u}{d x}$ is absolutely continuous in every closed subinterval $[a, b]$ $(0<a<b<\infty)$ in $(0, \infty)$.

iv) $u(x)$ has a compact carrier in $(0, \infty)$.

v) $-\frac{d^{2} u}{d^{2} x}+q(x) u(x) \in \mathrm{L}_{2}(0, \infty ; d x)$.

Define an operator $L_{\infty}$ which transforms $u(x) \in \mathscr{D}_{\infty}$ to

$$
L_{\infty} u(x)=-\frac{d^{2} u}{d x^{2}}+q(x) u(x) .
$$

By the assumption of Theorem 1, if we denote the closure of $L_{\infty}$ by $L, L$ is 
a self-adjoint operator. Let $l$ be a complex number with $I_{m} l \neq 0$ and $\boldsymbol{L}_{l}$ be the resolvent $(l-L)^{-1}$. We have for $u(x)$ in $\mathscr{D}_{\infty}$

$$
\mathscr{T}_{P_{k}}(l-L) u(\lambda)=(l-\lambda) \mathscr{T}_{P_{k}} u(\lambda), \quad(k=1,2) .
$$

Therefore we obtain

$$
\begin{aligned}
\left\langle\boldsymbol{L}_{l}(l-L) u, f\right\rangle & =\langle u, f\rangle=\left\langle\mathscr{T}_{\boldsymbol{P}_{\boldsymbol{k}}} u, \mathscr{I}_{\boldsymbol{P}_{\boldsymbol{k}}} f\right\rangle_{\boldsymbol{P}_{\boldsymbol{k}}} \\
& =\left\langle\frac{\mathscr{T}_{\boldsymbol{P}_{\boldsymbol{k}}}(l-L) u}{l-\lambda}, \mathscr{I}_{\boldsymbol{P}_{\boldsymbol{k}}} f\right\rangle_{\boldsymbol{P}_{\boldsymbol{k}}}, \quad(k=1,2)
\end{aligned}
$$

for $u(x)$ in $\mathscr{D}_{\infty}$ and $f(x)$ in $\mathrm{L}_{2}(0, \infty ; d x)$. Since the family of functions $\left\{(l-L) u(x) / u(x) \in \mathscr{D}_{\infty}\right\}$ is dense in $\mathrm{L}_{2}(0, \infty ; d x)$, we have

$$
\left\langle\boldsymbol{L}_{l} f, h\right\rangle=\int_{-\infty}^{\infty} \frac{\tilde{\mathscr{T}}_{\boldsymbol{P}_{k}} f(\lambda) d \boldsymbol{P}_{\boldsymbol{k}}(\lambda) \overline{\mathscr{I}_{\boldsymbol{P}} h}(\lambda)}{\lambda-l}, \quad(k=1,2)^{(1)}
$$

for every pair of $f(x)$ and $h(x)$ in $\mathrm{L}_{2}(0, \infty ; d x)$.

Let $E_{P_{1}}(\Delta)$ and $E_{P_{2}}(\Delta)$ be the operators in Lemma 1 with respect to $\boldsymbol{P}_{1}(\Delta)$ and $\boldsymbol{P}_{2}(\Delta)$. Then making use of the inversion formula for Stieltjes transformation ${ }^{(2)}$ we have from (1.20)

$$
\left\langle E_{P_{1}}(\Delta) f, h\right\rangle=\left\langle E_{P_{2}}(\Delta) f, h\right\rangle
$$

for every finite interval $\Delta$ in $(-\infty, \infty)$. From (1.21) and (1.5) we get

$$
E_{P_{1}}(x, y ; \Delta)=E_{P_{2}}(x, y ; \Delta),
$$

namely

$$
\int_{\Delta} \tilde{\boldsymbol{y}}(x, \lambda) d \boldsymbol{P}_{1}(\lambda) \boldsymbol{y}(y, \lambda)=\int_{\Delta} \tilde{\boldsymbol{y}}(x, \lambda) d \boldsymbol{P}_{2}(\lambda) \boldsymbol{y}(y, \lambda) .
$$

Let $\boldsymbol{y}_{0}(x, l)$ be a system of solutions

$$
\boldsymbol{y}_{0}(x, l)=\left(\begin{array}{l}
\varphi_{1}^{(0)}(x, l) \\
\varphi_{2}^{(0)}(x, l)
\end{array}\right)
$$

such that

$$
\begin{cases}\varphi_{1}^{(0)}(1, l)=0, & \frac{\partial \varphi_{1}^{(0)}}{\partial x}(1, l)=1, \\ \varphi_{2}^{(0)}(1, l)=1, & \frac{\partial \varphi_{2}^{(0)}}{\partial x}(1, l)=0\end{cases}
$$

(1) This formula is due to M. Matsuda.

(2) Neumark [7], Anhang. 
Then there exists a matrix

$$
\boldsymbol{A}(l)=\left(\begin{array}{cc}
\alpha(l) & \gamma(l) \\
\beta(l) & \delta(l)
\end{array}\right), \quad \alpha, \beta, \gamma, \delta \text { being entire functions of } l
$$

such that

$$
\boldsymbol{y}(x, l)=\boldsymbol{A}(l) \boldsymbol{y}_{0}(x, l) .
$$

Define two density matrices $d \boldsymbol{P}_{1}^{(0)}(\lambda)$ and $d \boldsymbol{P}_{2}^{(0)}(\lambda)$ by

$$
d \boldsymbol{P}_{k}^{(0)}(\lambda)=\widetilde{\boldsymbol{A}(\lambda)} d \boldsymbol{P}_{k}(\lambda) \boldsymbol{A}(\lambda), \quad k=1,2 .
$$

Then the measure matrices $\boldsymbol{P}_{k}^{(0)}(\lambda)(k=1,2)$ will satisfy (A), (B) and (C) with respect to $\boldsymbol{y}_{0}(x, \lambda)$.

To prove $\boldsymbol{P}_{1}(\Delta)=\boldsymbol{P}_{2}(\Delta)$ it is sufficient to prove $\boldsymbol{P}_{1}^{(0)}(\Delta)=\boldsymbol{P}_{2}^{(0)}(\Delta)$. From (1.2), (1.25) and (1.26) we obtain for $k=1,2$

$$
E_{\boldsymbol{P}_{k}}(x, y ; \Delta)=\int_{\Delta} \tilde{\boldsymbol{y}}_{0}(x, \lambda) d \boldsymbol{P}_{k}^{(0)}(\lambda) \boldsymbol{y}_{0}(y, \lambda),
$$

and hence (1.22) implies

$$
\int_{\Delta} \tilde{\boldsymbol{y}}_{0}(x, \lambda) d \boldsymbol{P}_{1}^{(0)}(\lambda) \boldsymbol{y}_{0}(y, \lambda)=\int_{\Delta} \widetilde{\boldsymbol{y}}_{0}(x, \lambda) d \boldsymbol{P}_{2}^{(0)}(\lambda) \boldsymbol{y}_{0}(y, \lambda) .
$$

We differentiate (1.27) with respect to $x$ or $y$ to obtain

$$
\int_{\Delta} \frac{\partial \tilde{\boldsymbol{y}}_{0}(x, \lambda)}{\partial x} d \boldsymbol{P}_{1}^{(0)}(\lambda) \boldsymbol{y}_{0}(y, \lambda)=\int_{\Delta} \frac{\partial \tilde{\boldsymbol{y}}_{0}(x, \lambda)}{\partial x} d \boldsymbol{P}_{2}^{(0)}(\lambda) \boldsymbol{y}_{0}(y, \lambda) .
$$

and

$$
\int_{\Delta} \frac{\partial \tilde{\boldsymbol{y}}_{0}(x, \lambda)}{\partial x} d \boldsymbol{P}_{1}^{(0<}(\lambda) \frac{\partial \boldsymbol{y}_{0}(y, \lambda)}{\partial y}=\int_{\Delta} \frac{\partial \tilde{\boldsymbol{y}}_{0}(x, \lambda)}{\partial x} d \boldsymbol{P}_{2}^{(0)}(\lambda) \frac{\partial \boldsymbol{y}_{0}(y, \lambda)}{\partial y}
$$

Set

$$
\boldsymbol{P}_{k}^{(0)}(\Delta)=\left(\begin{array}{ll}
\rho_{11}^{(k)}(\Delta) & \rho_{12}^{(k)}(\Delta) \\
\rho_{21}^{(k)}(\Delta) & \rho_{22}^{(k)}(\Delta)
\end{array}\right), \quad(k=1,2) .
$$

Then putting $x=y=1$ in (1.27), (1.28) and (1.29) we have

$$
\rho_{22}^{(1)}(\Delta)=\rho_{22}^{(2)}(\Delta), \quad \rho_{22}^{(1)}(\Delta)=\rho_{12}^{(2)}(\Delta), \quad \rho_{11}^{(1)}(\Delta)=\rho_{11}^{(2)}(\Delta)
$$

respectively, which completes the proof.

Proof of Theorem 2. Let $\boldsymbol{P}(\lambda)$ be a measure matrix which satisfies (A) and (B). Then we have 


$$
\left\langle\boldsymbol{L}_{l} f, h\right\rangle=\int_{-\infty}^{\infty} \frac{\tilde{\mathscr{I}}_{\boldsymbol{P}} f(\lambda) d \boldsymbol{P}(\lambda) \overline{\mathscr{F}_{\boldsymbol{P}}} h(\lambda)}{l-\lambda},
$$

and $E_{P}(\Delta)$ becomes a resolution of the identity ${ }^{(1)}$.

Let $\boldsymbol{y}_{0}(x, \lambda)$ be a system of solutions which satisfies the initial conditions (1.24). Putting

$$
d \boldsymbol{P}_{0}(\lambda)=\tilde{\boldsymbol{A}}(\lambda) d \boldsymbol{P}(\lambda) \boldsymbol{A}(\lambda)
$$

for $\boldsymbol{A}(l)$ satisfying (1.25), we have a resolution of the identity $E_{P_{0}}(\Delta)$.

Defining $\boldsymbol{u}_{0}(x, \Delta)$ by

$$
\boldsymbol{u}_{0}(x, \Delta)=\int_{\Delta} d \boldsymbol{P}_{0}(\lambda) \boldsymbol{y}_{0}(x, \lambda)=\left(\begin{array}{l}
u_{1}^{(0)}(x, \Delta) \\
u_{2}^{(0)}(x, \Delta)
\end{array}\right),
$$

we shall prove

$$
\boldsymbol{P}_{0}\left(\Delta \cap \Delta_{1}\right)=\int_{0}^{\infty} \boldsymbol{u}_{0}(x, \Delta) \tilde{\boldsymbol{u}}_{0}\left(x, \Delta_{1}\right) d x
$$

for every pair of intervals $\Delta$ and $\Delta_{1}$.

Since $E_{P_{0}}(\Delta)$ is a resolution of the identity, we have

$$
\int_{0}^{\infty} E_{P_{0}}(s, x ; \Delta) E_{P_{0}}\left(s, y ; \Delta_{1}\right) d s=E_{P_{0}}\left(x, y ; \Delta \cap \Delta_{1}\right) .
$$

By (iii) of Lemma 1 we can differentiate (1.33) with respect to $x$ or $y$ to obtain

$$
\int_{0}^{\infty} \frac{\partial E_{P_{0}}(s, x ; \Delta)}{\partial x} E_{P_{0}}\left(s, y ; \Delta_{1}\right) d s=\frac{\partial E_{P_{0}}\left(x, y ; \Delta \cap \Delta_{1}\right)}{\partial x},
$$

and

$$
\int_{0}^{\infty} \frac{\partial E_{P_{0}}(s, x ; \Delta)}{\partial x} \frac{\partial E_{P_{0}}\left(s, y ; \Delta_{1}\right)}{\partial y} d s=\frac{\partial^{2} E_{P_{0}}\left(x, y ; \Delta \cap \Delta_{1}\right)}{\partial x \partial y} .
$$

Setting $x=y=1$ in (1.33) and (1.34) and (1.35), we have

$$
\int_{0}^{\infty} u_{i}^{(0)}(s, \Delta) u_{j}^{(0)}\left(s, \Delta_{1}\right) d s=\rho_{i j}^{(0)}\left(\Delta \cap \Delta_{1}\right), \quad(i, j=1,2),
$$

where

$$
\boldsymbol{P}_{0}(\Delta)=\left(\begin{array}{ll}
\rho_{11}^{(0)}(\Delta) & \rho_{12}^{(0)}(\Delta) \\
\rho_{21}^{(0)}(\Delta) & \rho_{22}^{(0)}(\Delta)
\end{array}\right)
$$

(1) See the proof of Theorem 1 of Matsuda [6]. 
Thus the identity (1.32) is proved.

For $\boldsymbol{y}(x, l)$ and $\boldsymbol{P}(\Delta)$ putting

$$
\boldsymbol{u}(x, \Delta)=\int_{\Delta} d \boldsymbol{P}(\lambda) \boldsymbol{y}(x, y)
$$

we have by (1.36)

$$
\boldsymbol{P}\left(\Delta \cap \Delta_{1}\right)=\int_{0}^{\infty} \boldsymbol{u}(x, \Delta) \tilde{\boldsymbol{u}}\left(x, \Delta_{1}\right) d x .
$$

Define a transformation $\mathscr{F}_{\boldsymbol{P}}^{*}$ from $\mathrm{L}_{2}(-\infty, \infty ; d \boldsymbol{P}(\lambda))$ onto $\mathrm{L}_{2}(0, \infty$; $d x)$ by

$$
\mathscr{F}_{\boldsymbol{P}}^{*}: \boldsymbol{v}(\lambda) \rightarrow \int_{-\infty}^{\infty} \boldsymbol{y}(x, y) d \boldsymbol{P}(\lambda) \boldsymbol{v}(\lambda) .
$$

Then $\mathscr{F}_{\boldsymbol{P}}^{*} \cdot \mathscr{I}_{\boldsymbol{P}}$ proves to be the identity operator. ${ }^{(1)}$

By (1.38), we can prove that $\mathscr{F}_{\boldsymbol{P}}^{*}$ is an isometric transformation from $\mathrm{L}_{2}(-\infty, \infty ; d \boldsymbol{P}(\lambda))$ onto $\mathrm{L}_{2}(0, \infty ; d x)$ (Kodaira [3] 2, [5]). $\mathscr{I}_{\boldsymbol{P}}$ is therefore surjective, and the proof is completed.

Remark. If we assume the existence of the measure matrix $\boldsymbol{P}_{*}(\lambda)$ which satisfies (A), (B) and (C), calculated by Titchmarsh-Kodaira's spectral formula, the proof of Theorem 2 will be easier (Kodaira [3], [4]).

In fact, let $\boldsymbol{P}(\lambda)$ be a measure matrix satisfying (A) and (B). Then we have

$$
\left\langle\boldsymbol{L}_{l} f, h\right\rangle=\int_{-\infty}^{\infty} \frac{\mathscr{I}_{\boldsymbol{P}_{*}} f(\lambda) d \boldsymbol{P}_{*}(\lambda) \mathscr{I}_{\boldsymbol{P}_{*}} h(\lambda)}{l-\lambda}=\int_{-\infty}^{\infty} \frac{\mathscr{F}_{\boldsymbol{P}} f(\lambda) d \boldsymbol{P}(\lambda) \mathscr{I}_{\boldsymbol{P}} h(\lambda)}{l-\lambda} .
$$

Using Lemma 1 we have

$$
E_{P_{*}}(x, y ; \Delta)=E_{P}(x, y ; \Delta) .
$$

We obtain $\boldsymbol{P}_{*}(\Delta)=\boldsymbol{P}(\Delta)$ by the method used in the proof of Theorem 1 . Therefore $\boldsymbol{P}(\Delta)$ satisfies $(\mathrm{C})$.

If the equation (1.1) is of the linit cirdle type at 0 , the situation is essentially the same as in the case where $x=0$ is a regular point.

\section{§. The spectrum in the limit circle case at infinity.}

In the case where $x=0$ is a regular point of the equation (1.1),

(1) See Proposition 1 of Matsuda [6]. 
M. Matsuda has proved that the spectrum is unbounded below in the limit circle case at $x=\infty$ (M. Matsuda [6]).

In this section we assume that the equation (1.1) belongs to the limit circle case at $x=\infty$. Then by setting some boundary conditions at $\infty$ and also at 0 if necessary, we obtain a self-adjoint operator $L$ which is a symmetric extension of the $L_{\infty}$ in $\S 1$. The spectrum of this operator is simple ${ }^{(1)}$.

Then we shall prove the following theorem:

Theorem 3. Let the equation (1.1) belong to the limit circle case at $\infty$. Then the self-adjoint operator $L$ is unbounded below.

In fact, let $I_{1}=[1, \infty)$ and $I_{2}=(0,1]$. Setting some boundary conditions at $x=1$, we obtain $L_{1}$ and $L_{2}$ which are the restricitions of $L$ to $I_{1}$ and to $I_{2}$ respectively. Then $L$ is bounded below if and only if $L_{1}$ and $L_{2}$ are both bounded below ${ }^{(2)}$. $L_{1}$ is unbounded below by virtue of Theorem 2 of Matsuda [6], and hence $L$ is also unbounded below.

Using Weyl's classification of the limit point case and the limit circle case, we can see that Theorem 3 is equivalent to the following fact:

Let $q(x)$ be locally summable in $(0, \infty)$. Then if $L_{\infty}$ in $\S 1$ is bounded below, $L_{\infty}$ is essentially self-adjoint.

Let us make a remark on this fact. In the $m$-dimensional case, the following result is known (Wienholtz [8], Kato [2]):

Let $L_{0}$ be a partial differential operator

$$
L_{0}=-\Delta+q(x),
$$

where $q(x)$ has a following property: there exists a constant $\alpha(0<\alpha<1)$ such that

$$
M(x)=\int_{|x-y| \geqq 1}|x-y|^{\mu(m, a)}|q(y)|^{2} d y, \quad \mu(m, \alpha)= \begin{cases}0, & m \leqq 3 \\ -m+4-\alpha, & m \geqq 4\end{cases}
$$

is locally bounded. The domain of $L_{0}$ consists of $C^{\infty}$-functions of compact carrier. Then if $L_{0}$ is bounded below, $L_{0}$ is essentially self-adjoint.

By slight modification of their method we can replace the local

(1) Kodaira [3].

(2) Dunford-Schwartz [1]. p. 1455. 
boundedness of $M(x)$ with the local summability of $q(x)$ to prove the fact we obtained above. However, our method seems to be of some interest in that we derived this in the scheme of the inverse problem of GelfandLevitan.

\section{BIBLIOGRAPHY}

[1] Dunford, N. and J. Schwartz, Linear Operators, part II, Interscience, New York (1963).

[2] Kato, T., Partial differential equations in quantum mechanics, Sugaku 10, (1958) 212-219 (Japanese).

[ 3 ] Kodaira, K., On singualr solutions of second-order differential operators, Sugaku 1 (1948); 2 (1949), 113-139 (Japanese).

[4] Kodaira, K., The eigenvalue problem for ordinary differential equations of the second order and Heisenberg's theory of S-matrix, Amer. J. Math. 71 (1949) 921-945.

[5] Kodaira, K., On ordinary differential equations of any even order and the corresponding eigenfunction expansions, Amer. J. Math. 72 (1950), 502-544.

[6] Matsuda, M., Orthogonality of generalized eigenfunctions in Weyl's expansion theorem, Publ. of Research Institute for Mathematical Sciences, Kyoto University, Ser. A, 2 (1966), 243-254.

[7] Neumark, M.A., Lineare Differentialoperatoren, Akademie-Verlag, Berlin, (1960).

[8] Wienholtz, E., Halbbeschrankte partielle Differentialoperatoren zweiter Ordnung vom elliptischen Typus, Math. Ann. 135 (1958), 50-80. 
